\title{
Toward an Integrated History and Philosophy of Diagrammatic Practices
}

\author{
Chiara Ambrosio \\ Department of Science and Technology Studies
}

$\mathrm{UCL}$

\author{
c.ambrosio@ucl.ac.uk
}

\begin{abstract}
This article offers an overview of current approaches to the study of diagrams and their roles in scientific knowledge-making. The discussion develops in three parts. The first part investigates and questions historical and philosophical analyses of the suppression of diagrams in the nineteenth and twentieth centuries. It attempts to sketch an alternative historiography of diagrammatic practices, in which the insights of advocates of diagrammatic reasoning in a time of "objectivity without images" take center stage. The second part of the article turns to the American philosopher, scientist and logician Charles Sanders Peirce, as a representative defender of diagrammatic reasoning and diagrammatic representation in the late nineteenth century, and investigates his legacy on current approaches to diagrams. The third and final part exposes a puzzling paradox in the literature, characterizing it as a false dichotomy between "the representational view" and the "object-

based view" of diagrams. The article concludes that this dichotomy reveals more about the identities of scholars embracing particular disciplinary traditions than about diagrams themselves, and suggests that it can be overcome by attending to diagramming as a practice at the intersections of representation, manipulation and experimentation.
\end{abstract}

Diagrams have steadily gained the attention of scholars across history, philosophy, and social studies of science in recent years, to the point that their constitutive role in the production, validation and dissemination of scientific knowledge seems today fairly uncontentious. As this special issue shows, the sustained study of the practice of diagramming casts light on aspects of scientific knowledge-making as varied as representing, configuring and using evidence (Morgan), capturing values and moral commitments (Maas), mastering uncertainty (Boumans), tracing procedural sequences for the purpose of transmitting and using technical knowledge (Bray), modelling and idealisation (Chao), and drawing new historiographical implications from the diagrammatic form of ancient texts (Chemla). This variety of approaches reflects the plurality of purposes for which diagrams are produced and used in a range of scientific practices and across cultural, historical and geographical contexts. But embracing this variety as a defining feature of the roles and functions of diagrams in knowledge-making has been itself an achievement of the disciplines and scholars involved in defending it as a virtue, rather than a hindrance, in the production of scientific knowledge.

This critical overview of the current state of the study of diagrams has three main goals. First, I assess and question various historiographical analyses of the suppression of diagrams in the nineteenth and early twentieth century. This body of literature attributes the 'eclipse' of diagrams to foundational debates especially in mathematics and logic, and ascribes their reappearance in the second half of the twentieth century to a broader shift toward scientific practice and toward the visual and material aspects of knowledge production in the sciences. Contrary to this dominant view, I aim to show that, foundational debates notwithstanding, diagrams never really disappeared from scientific practice. In fact, it is by paying attention to advocates of diagrammatic reasoning and diagrammatic representations precisely in this period that the recent scholarship has developed new conceptual tools to analyse the diverse roles this kind of representations play in scientific knowledge-making. 
One such advocate was the American logician, scientist, and philosopher Charles Sanders Peirce, whom I single out as a case study in the second part of this review. For one thing, Peirce was a champion of diagrammatic reasoning in practice, as well as in theory. 'I do not think I ever reflect in words', he famously reminisced in 1909, 'I employ visual diagrams firstly because this way of thinking is my natural language of self-communion, and secondly because I am convinced that it is the best system for the purpose' (R 619: 8, 1909). ${ }^{1}$ Indeed, since at least the 1960s the study of Peirce's Existential Graphs, a diagrammatic system which he formally put forward in 1896, has been instrumental in the development of a distinctive strand of logic aimed at vindicating the legitimacy of non-linguistic representation in inference and reasoning. But Peirce was also one of the founding fathers of semiotics, and it is in fact one of his semiotic categories - iconicity - which is now invoked in most studies of diagrammatic representations. More broadly, the revival and systematic study particularly of Peirce's logical and semiotic writings has now produced an important body of literature with a still unfulfilled potential to contribute to general discussions of diagrams in history and philosophy of science; it is this potential which I hope to highlight and articulate in the central part of this review.

Thirdly and finally, I aim to expose a curious paradox in the literature. I characterise it (in a slightly artificial way, only to show its limitations) as a false dichotomy between the 'representational view' and the 'object-based view' of diagrams. ${ }^{2}$ While logicians and some philosophers unproblematically refer to diagrams as 'representations', the renewed attention to scientific practice and material culture in history and sociology of science, as well as in the now consolidated field of visual culture, has resulted in underplaying the representational aspects of diagrams to privilege instead their function as tools, artefacts or material objects in their own right. I will show that it is not entirely clear what conception of representation is the enemy here, and why embracing a historical, practice-based approach should entail dispensing with the representational aspects of diagrams altogether. Drawing on conceptual resources from historical epistemology, I will demonstrate that the false dichotomy between the representational view and the object-based view tells us more about the identities of scholars embracing particular disciplinary, epistemological, and historical traditions, than about diagrams themselves.

I will conclude with a plea for an integrated history, philosophy and visual culture of diagrammatic representations in practice. The strength of diagrams as representations, investigative tools and objects of inquiry in their own right is precisely, I will argue, in the fact that they inhabit a conceptual space at the intersection of disciplines - and as such they offer a fertile ground for an integrated, interdisciplinary approach to knowledge-making in the sciences.

\section{The Eclipse of Diagrams?}

It is often pointed out in the scholarly literature that diagrams resurfaced in philosophical discourse only in the second half of the twentieth century, after a period of prolonged scepticism. Foundational debates in nineteenth-century mathematics and logic are commonly recognised as the historical foil against which this revival took place. ${ }^{3}$ As Marcus Giaquinto $(2007 ; 2016)$ and Paolo

\footnotetext{
${ }^{1}$ I follow the convention of citing Peirce's unpublished manuscripts by manuscript number and page, as assigned in Robin (1967).

${ }^{2}$ Mary Morgan in this special issue also notes this tension and explicitly rejects it - see her footnote no.1

${ }^{3}$ I am grateful to Harro Maas for alerting me to how different the situation was in fields such as economics. Klein (1997), for instance, situates the suppression of diagrams in economics in the first part of the nineteenth
} 
Mancosu (2005) note, a broader suspicion toward the reliability of visual thinking emerged as a result of the realisation that geometric and spatial intuitions, which had a prominent place in Kantian epistemology of mathematics, turned out to produce unwarranted mathematical claims. Many of the epistemological questions philosophers now ask about the nature and role of diagrams in a broad range of contexts, even beyond foundational issues in mathematics and logic, were substantively shaped by the rippling effects of the terms in which these debates were cast in the nineteenth century. One example is the divide drawn between what philosophers would now label as the context of discovery and the context of justification. In a much-quoted passage from his influential Vorlesungen über neuere Geometrie (Lectures in Modern Geometry, 1882), the mathematician Moritz Pasch presented a characterisation of this divide which became a standard reference in the decades to follow:

'For the appeal to the figure is, in general, not at all necessary. It does facilitate essentially the grasp of the relations stated in the theorem and the constructions applied in the proof. Moreover, it is a fruitful tool to discover such relationships and constructions. However, if one is not afraid of the sacrifice of time and effort involved, then one can omit the figure in the proof of any theorem; indeed, the theorem is only truly demonstrated if the proof is completely independent of the figure' (translated and cited in Mancosu 2005, 14).

Pasch's attitude is distinctive of a broader approach emerging in foundational debates in the fields of analysis, calculus, and even in geometry: while used as psychological, heuristic aids, diagrams and figures came to be considered as dispensable in rigorous proof, which should instead be carried out through purely formal means. The most extreme positions in this debate drew even stronger conclusions about visualisation and its limitations: diagrams were not just dispensable - they should actively be avoided as a source of error when it came to the justification of mathematical claims. Rigorous proof, in turn, was recast as a sequence of sentences, each either an assumption of the proof, or derived via sound logical rules from previous sentences (Mumma 2010, 255). Vestiges of this more radical version of 'anti-visual puritanism' (Giaquinto 2008, 23) persisted in logical and philosophical quarters well into the second half of the twentieth century: 'The proof', wrote Neil Tennant in a 1986 article, 'is a syntactic object consisting only of sentences arranged in a finite and inspectable array' (Tennant 1986, 304).

Philosophers have traced a lineage, primarily via Gottlob Frege, David Hilbert and Bertrand Russell, from these foundational debates in geometry and analysis to logic, and via logic to epistemology and philosophy of science, especially in the Anglo-American tradition. Cathy Legg $(2013,1)$, for instance, points out how 'th[e] arguably contingent state of affairs' of nineteenthcentury debates in the foundations of mathematics contributes to explain the neglect of diagrams and diagrammatic reasoning in twentieth-century analytical philosophy, particularly as far as theorising about semantic content and the epistemological status of proof are concerned. Strict formalism was the means through which, for example, the logical positivists recast the task of philosophy as conceptual analysis, drawing a sharp divide between analytic and synthetic statements. The distinction left no space precisely for those portions of scientific activity - such as diagramming, but also importantly modelling - which seemed to fall outside its boundaries. Mathematics and logic fall within the realm of analytic knowledge, in this view: their claims are devoid of empirical content and merely record the conventions regulating the rigorous use of

century, in conjunction with the discrediting of William Playfair's work. Maas and Morgan (2002) connect the resurfacing of graphical methods in British political economy at the end of the nineteenth century to a broader shift in attitudes toward history and statistics - I will return to their work later on in this section. 
symbolic formalism. Synthetic knowledge, on the other hand, comprises claims whose factual content can be assessed empirically. Sentences like 'metals expand when heated' are synthetic - and meaningful, in the logical positivists' view - because they offer specific and syntactically well-formed 'empirical hypotheses' (Ayer, 1953; Carnap [1932] 1959), for which there are (or at least this was the hope of logical positivists in their early verificationist phase) clearly identifiable methods of verification by means of observation. 'It is hard to see' Legg $(2013,3)$ reminds us, 'how a diagram could offer an empirical hypothesis' in this strictly empiricist sense. It is equally hard to see how a diagram may fall within the realm of analytic knowledge, precisely in light of the dubious status of spatial intuitions that logical positivists had inherited from earlier debates in the foundations of mathematics.

In their history of scientific objectivity, Lorraine Daston and Peter Galison (2007) draw an analogous lineage, and contend that the dominance of formalism over images in this particular historical period is best contextualised as an expression of the epistemic virtue they dubbed 'structural objectivity'. 'There is a form of objectivity', they claim, 'that spurns all images, whether they are perceived by the eye of the body or that of the mind, as irretrievably subjective' (Daston and Galison 2007, 254). At the core of structural objectivity was a more general attempt at breaking away from the contingency and variability of private experience and individual sensations:

'The objective was not what could be sensed or intuited, for sensations and intuitions could be shown to differ, and in ways that were incorrigibly private for each person. Nor was it the bare face of facts, scrubbed free of any theoretical interpretation, for today's facts might be cast in a wholly different light by tomorrow's findings. Objectivity, according to the structuralists, was not about sensation or even about things; it had nothing to do with images, made or mental. It was about enduring structural relationships that survived mathematical transformations, scientific revolutions, shifts of linguistic perspective, cultural diversity, psychological evolution, the vagaries of history, and the quirks of individual physiology' (Daston and Galison 2007, 259).

Going beyond foundational debates in mathematics and logic, and acknowledging that the very meaning of 'structure' varied between historical actors, ${ }^{5}$ Daston and Galison suggest that even the field of psychology - eschewed by advocates of rigorous formal proof as the very stronghold of subjectivity ${ }^{6}$ - underwent a shift toward the primacy of structures as the main conduit to stable, invariable, and universally communicable relations. The approach to sensory physiology advocated by Herman von Helmholtz is a case in point. In Daston and Galison's account, Helmholtz's theory of unconscious inferences - the view that sensations are symbols, and not mere 'copies', of their corresponding stimuli - was motivated by a quest to render inscrutably private phenomena

\footnotetext{
${ }^{4}$ The reception of Daston and Galison's work has strangely overlooked their category of structural objectivity, mainly focusing on 'truth-to-nature', the attempt to idealise from instances in nature that preceded the establishment of the very notion of objectivity (Daston and Galison, 2007, chapter 2), and especially on 'mechanical objectivity', the representative ideal that preceded structural objectivity and consisted mainly of 'letting nature speak by itself' (Daston and Galison 2007, 120). See for example Jardine (2011) and Pickstone (2009). Even Boumans (2016), who specifically focuses on graphs in assessing the role of economists' expert judgment in inductive inferences, ultimately chooses mechanical objectivity as the foil of his argument (Boumans 2016, 4).

${ }^{5}$ Indeed, as Leo Corry (1996) has demonstrated, even the strictly mathematical formulation of 'structure' was contested at this point.

${ }^{6}$ Here the rejection of the visual as merely 'psychological' crosses paths with the much broader debate on psychologism, on which see Kusch 1995.
} 
amenable to empirical investigation. Interpreting sensations as abstract linguistic symbols, rather than images of the outer world, would open the possibility to pinpoint invariant relations among them - and therein lay the possibility of an objective science of the mind.

But while Helmholtz's account of unconscious inference might lend itself to an interpretation in terms of structural objectivity, his early experiments on the velocity of nerve impulses tell a different story. A distinctive dimension of the psychophysiological approach advocated by Helmholtz and his followers was time: ${ }^{7}$ that nerve transmission and reactions to stimuli had a temporality rendered these phenomena amenable to measurement. In a spirit that expanded Helmholtz's programme to ground even geometry and arithmetic in experience, Wilhelm Wundt argued that the very conception of number originated from the intuition of time, itself consisting of the measurable succession of sensations in consciousness. 'Brandishing stopwatch and metronome', Daston and Galison claim, 'experimental psychology took up Helmholtz's challenge to anchor number concepts in experience' (Daston and Galison 2007, 265).

Yet, at this very junction of psychology and mathematics lies a paradox of structural objectivity, a paradox which revolves precisely around the contested epistemological status of diagrams in these debates. As Kathryn Olesko and Frederic Holmes (1995) have argued, Helmholtz's experimental inquiries centrally revolved around graphical representations, which provided an 'image of precision' with an enduring legacy in nineteenth and early twentieth-century life sciences. The graphical imagery of these experiments was itself rooted in a broader context: 'the spreading usage of graphical methods of recording and presenting data at just this time', Olesko and Holmes point out, 'suggests that Helmholtz was attuned to contemporary trends that would in the future continually reinforce the expository power of his graphical method for determining the velocity of nerve impulse' (Holmes and Olesko 1995, 217). Taking this line of investigation even further, Robert Brain and M. Norton Wise (1994) have demonstrated, in what is now a classic article, that Helmholtz's own graphical representations of the propagation speed of stimulation in nerves were in turn grounded in the broader visual and technical culture of indicator diagrams, originally used by James Watt to measure and diagnose the work performed in the cylinder of a steam engine (cf. also Wise 2018). And as Henning Schmidgen (2014) has shown, this line of investigation invites an interpretation of Helmholtz's time experiments as characterised by a distinctively semiotic, material, and ultimately diagrammatic dimension. While Helmholtz's curves plotted the temporality of nerve reactions visually, numerical values lent formal accuracy to those measurements. With a nod to Galison (1997), Schmidgen observes: 'Image and logic, icon and number, Bild and Zahl, therefore, do not yet belong to separate traditions, as in the laboratory practice of physicists involved in big science. Instead, they figure as closely related aspects of a single experimental process in the emerging field of physiology' (Schmidgen 2014, 9).

What the case of Helmholtz seems to suggest is that perhaps structural objectivity separates too neatly forms of knowledge-making that in practice were far more intertwined, and whose boundaries were constantly negotiated - by scientists, mathematicians, and logicians alike. Indeed, the century of 'anti-visual' puritanism and 'objectivity without images' turns out to be also the century in which the word 'graph' was first introduced in the English language by the mathematician James Joseph Sylvester, who used it in 1878 to describe the striking similarity that graphical

\footnotetext{
${ }^{7}$ See Schmidgen 2014 for an overview of reaction time measurements and the notion of 'temps perdu' in Helmholtz.
} 
representations of invariants and covariants of binary quantics exhibited with chemical bonds in molecules (Sylvester 1878; Hankins 1999; Hankins and Silverman 1995; see fig 1).

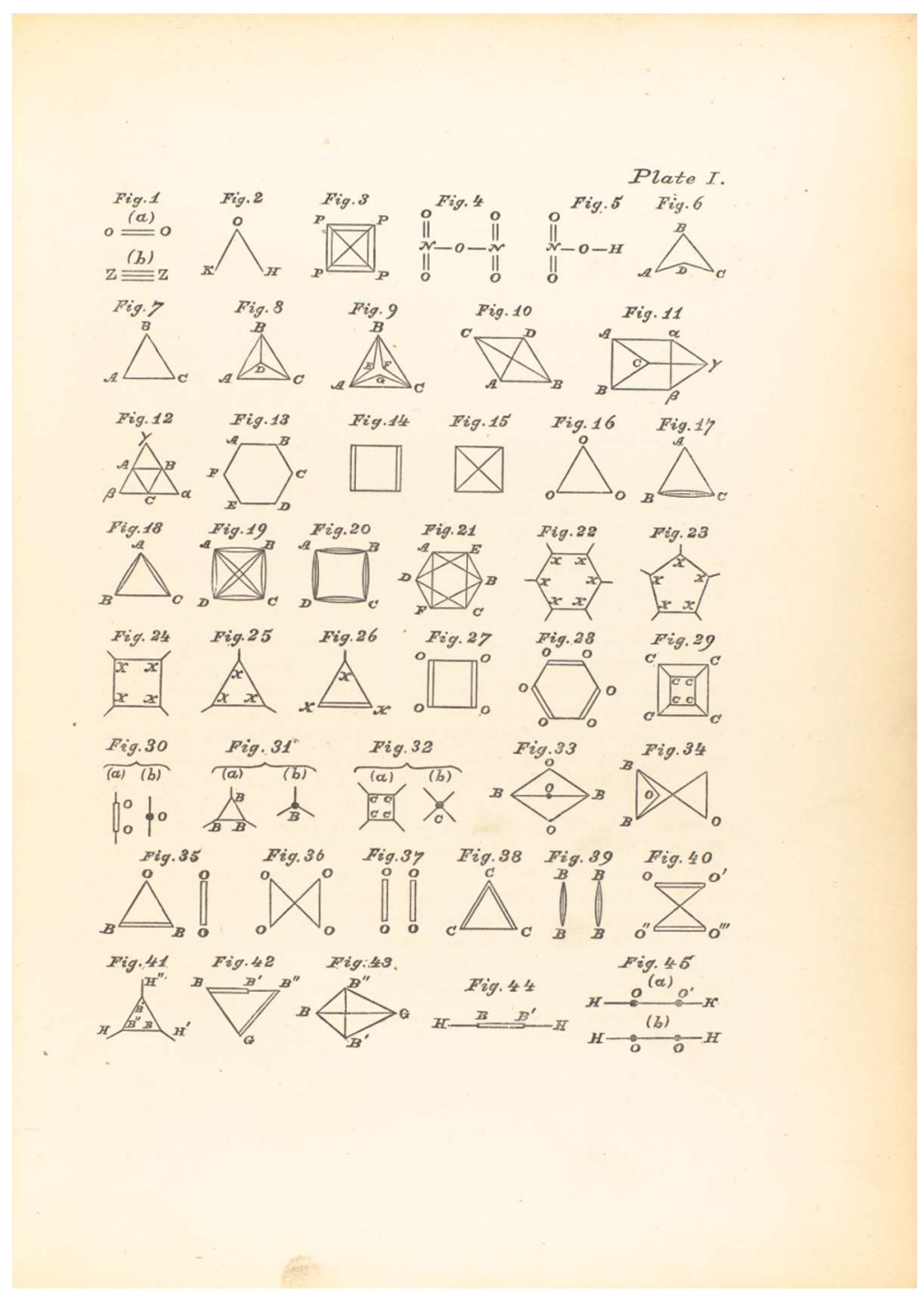

Fig. 1. Sylvester's Graphs. From Sylvester (1878), facing page 82. Courtesy of UCL Special Collections.

The meaning Sylvester attributed to his chemical-algebraic diagrams persisted in the branch of mathematics now known as 'graph theory' (Biggs, Lloyd Wilson 1976). On the other hand, as Thomas Hankins (1999; 2006; cf. also Hankins and Silverman 1995, 141) has shown, it was only by the beginning of the twentieth century that the term 'graph' came to define a different kind of diagrammatic representations, which related two measured quantities and their respective changes by means of a curve - precisely as in Helmholtz's graphical experiments. In The Philosophy of the Inductive Sciences, the British polymath William Whewell referred to this particular use of graphical representations as the 'Method of Curves', presenting it as one of the 'special methods' of induction, through which general laws in the sciences could be obtained from observations. In aphorism XLIV, he states: 
'The Method of Curves consists in drawing a curve, of which the observed quantities are the Ordinates, the quantity on which the change of these quantities depends being the Abscissa. The efficacy of this Method depends upon the faculty which the eye possesses of readily detecting regularity and irregularity in forms. The Method may be used to detect the Laws which the observed quantities follow; and also, when the Observations are inexact, it may be used to correct these Observations, so as to obtain data more true than the observed facts themselves' (Whewell 1858, 202)

Whewell singled out two advantages of the Method of Curves. For one thing, graphs allowed scientists to grasp the relationship between observational data, in a way that numerical tables could not afford. Referencing his own tidal researches, Whewell gave a vivid description of how the scientist's eye discovered order by following the line of a graph:

'If these Numbers [i.e. on tide tables] are expressed by the magnitude of Lines, and if these Lines are arranged in regular order, the eye discovers the rule of their changes: it follows the curve which runs along their extremities and takes note of the order in which the convexities and concavities succeed each other, if any order be readily discoverable' (Whewell 1858, 205).

Hankins $(2006,618)$ notes that the Method of Curves is in line with Whewell's account of the crucial role of ideas in induction. Indeed, inductive inference for Whewell is not exhausted by the simple enumeration, juxtaposition or combination of observed facts. Instead, it involves an active contribution by the mind: 'There is a New Element added to the combination by the very act of thought by which they are combined. There is a Conception of the mind introduced in the general proposition which did not exist in any of the observed facts' (Whewell 1858, 72). Thus, the first advantage of the Method of Curves is that it makes the process of introducing a new idea visible. As Hankins notes: 'A graph allowed the mind to 'see' the general idea in a set of data and impose the necessary mental superinduction to arrive at a scientific law' (Hankins 2006, 618).

A second advantage of the method of curves is that according to Whewell it accounted for the possibility of deriving general laws from imperfect observations. When imperfect observations are plotted on a graph, the resulting curve is 'broken and irregular...full of sudden and capricious twistings' (Whewell 1858, 206). The scientist's task is to smooth out these random deviations, which are small in comparison to the general tendency of the curve:

'By this method, thus getting rid at once, in a great measure, of errors of observation, we obtain data which are more true than the individual facts themselves. The philosopher's business is to compare his hypotheses with facts...but if we make the comparison with separate special facts, we are liable to be perplexed or misled, to an unknown amount, by the errours [sic] of observation...if however we take the whole mass of the facts and remove the errours of actual observation, by making the curve which expresses the supposed observation regular and smooth, we have the separate facts connected by their general tendency. We are put in possession, as we have said, of something more true than any fact by itself is' (Whewell 1858, 207).

Whewell's extensive and detailed reflections on the Method of Curves show that graphical representations began acquiring methodological and epistemological importance precisely at the dawn of what Daston and Galison define as 'objectivity without images'. Indeed, his systematic methodological analysis of the virtues of graphical representations was part and parcel of the very pursuit of universal communicability that Daston and Galison place at the core of structural 
objectivity. For a less obvious point emerging from Whewell's account of the Method of Curves is that graphs make ideas and their role in inductive inferences more than just visible to the individual scientist: they make them public and communicable within an entire community. This aspect of Whewell's work has been noticed especially in accounts of the influence of the Method of Curves beyond the natural sciences. As Harro Maas and Mary Morgan (2002) and Marcel Boumans (2006) have noticed, Whewell's defence of the graphical method was crucial in setting the premises and methodological context for the use of graphs in support of inductive arguments in economics in the second half of the nineteenth century. What Whewell offered was an articulation of how the graphical method prompted external (as opposed to introspective) reflection: as Maas and Morgan explain, 'for Whewell...induction had nothing to do with internal reflection and everything to do with external reflection, the reflection of observations made over time 'in the clear mirror space' of the graph' (Maas and Morgan, 2001, 111). At the same time, his advocacy of graphs fitted within the broader history of objectivity, in that it offered a publicly ascertainable method to account for the role of expert judgment in the removal of bias. As Boumans observes: 'Whewell showed how this kind of judgment could be involved using the Method of Curves, that is by defining unbiasedness as a specific feature of a visual display of the data, namely smoothness, for which the 'eye' is an effective tool for assessment' (Boumans 2006, 4).

In a 2006 article entitled 'Making Visible', M. Norton Wise advocates a shift from 'dichotomies' to 'intersections' in theorising the range of scientific practices and activities that lay at the boundaries between art and science, museums and laboratories, algebra and geometry, and more broadly science and culture:

'The dichotomies of doing versus thinking, craftsperson versus creator of ideas, and body versus mind (or the senses versus the intellect) must then be transformed into overlapping actions, or intersections, where the 'and' of collaboration replaces the 'either/or' of intellectual conceit (Wise, 2008, 79).

The case of the emergence and consolidation of the graphical method in the second half of the nineteenth century is a compelling way in which diagrams count as materialisations of such overlapping actions. At the same time, the recent scholarship on the rise of graphical methods across the natural and social sciences in the nineteenth century helpfully disturbs linear narratives that cast formalisation, proof, the context of justification, and the quest for 'structures' as the winners of the day at the expenses of the visual - at least until images came back with a revenge in the second half of the twentieth century. It shows that - defence of images, diagrams, and visual thinking notwithstanding - these narratives still subscribe to the very dichotomy that diagrammatic representations by virtue of their heterogeneous nature defy.

Daston and Galison's argument about the pursuit of communicability as a distinctive mark of an ever-expanding community of scientists in the nineteenth century is many respects persuasive. Less persuasive is that the totality of this community deferred judgment exclusively to formal 'structures' as part of this pursuit, or more precisely that 'structures' were an expression of 'objectivity without images'. As the cases of Helmholtz and Whewell show, diagrams were part and parcel of empiricist accounts of knowledge that aspired - in different ways - to break out of the private world of sensations, intuitions, ideas, and introspection. In Helmholtz's case, it was the partly symbolic, partly visual nature of the graphical method that made phenomena as private as sensations measurable, publicly testable, and amenable to empirical investigation. For Whewell, the Method of Curves captured the intervention of ideas in grasping the laws underlying aggregates of 
observations despite their individual imperfections, at the same time exposing the process of 'smoothing' a curve to public scrutiny.

Helmholtz and Whewell were not alone in fighting their battle for universal communicability through diagrams. One of the strongest advocates of diagrammatic representations in the second half of the nineteenth century was the American philosopher Charles Sanders Peirce, whose writings form an important foundation for a range of contemporary approaches to diagrams. It is to Peirce and his legacy that I turn in the next section.

\section{Perspicuity, Communicability, Manipulability}

In an article entitled 'Prolegomena to an Apology of Pragmaticism', published in The Monist in 1906, Peirce describes the process of experimenting with diagrams through an intriguing imaginary dialogue with 'an eminent and glorious General' (CP 4.530). Peirce's fictional interlocutor expresses his desire to draw a map for the purpose of forecasting action on the battlefield: 'I might probably desire the maps to stick pins into, so as to mark each anticipated day's change in the situations of the two armies' (CP 4.530). Peirce's rejoinder clarifies the purpose of this imaginary exchange. The General is attributing to the map some important features that are distinctive of diagrams more broadly:

'Well, General, that precisely corresponds to the advantages of a diagram of the course of a discussion. Indeed, just there, where you have so clearly pointed it out, lies the advantage of diagrams in general. Namely...one can make exact experiments upon uniform diagrams; and when one does so, one must keep a bright lookout for unintended and unexpected changes thereby brought about in the relations of different significant parts of the diagram to one another. Such operations upon diagrams, whether external or imaginary, take the place of the experiments upon real things that one performs in Chemical and Physical research. Chemists have, ere now, I need not say, described experimentation as the putting of questions to Nature. Just so, experiments upon diagrams are questions put to the Nature of the Relations concerned'. (CP 4.530) ${ }^{8}$

Using pins on the map, Peirce's imaginary General would be able to reason upon, and investigate, the conceivable effects of the particular military configurations of two armies and their possible actions. In an analogous manner, experimentation with diagrams - even in the most abstract case of logical diagrams, which is what Peirce here has in mind - allows their users to reason upon logically possible, unintended and unexpected changes in the relations between the diagram's significant parts. This logical sense of 'experimentation' upon diagrams, Peirce continues, is not different from the kind of experimentation that occurs in the physical sciences, particularly chemical research. ${ }^{9}$

By 1906, Peirce had worked extensively with diagrams across mathematics, logic and the physical sciences. In 1869, the very year in which Dmitri Mendeleev's periodic table appeared in the German journal Zeitschrift für Chemie, he published an article titled 'The Pairing of the Elements' in the American edition of the journal Chemical News. In the article, Peirce proposed a diagrammatic

\footnotetext{
${ }^{8}$ Here ' $C P$ ' is the conventional abbreviation for the Collected Papers of Charles S. Peirce, in bibliography as Peirce (1931-8/1958).

${ }^{9}$ See Ambrosio and Campbell 2016 on the (much neglected) influence of chemistry and chemical education in Harvard on Peirce's account of diagrammatic representations.
} 
classification of the elements according to their atomic weight. ${ }^{10}$ The piece was praised by the American Association for the Advancement of Science as having 'greatly added to the illustration of the fact of pairing by representing in a diagram the elements in positions determined by ordinates representing atomic numbers' (W1, xx; fig. 2) ${ }^{11}$ Exactly ten years later, while simultaneously employed as an assistant for the US Coast and Geodetic Survey and as a lecturer in logic at Johns Hopkins University, Peirce presented a new system of 'quincuncial' map projection, based on a transformation of conformal stereographic projection, in the newly founded American Journal of Mathematics (fig 3; cf Kiryushchenko 2015; Ljungberg 2015; Kramer and Ljungberg 2016). ${ }^{12}$ Lastly, in 1896, Peirce put forward what he defined as his 'Chef d'Oeuvre': a diagrammatic system of logic which he labelled as his 'Existential Graphs' (fig. 4). ${ }^{13}$

\footnotetext{
${ }^{10}$ The article is reprinted in vol. 2 of the chronological edition of the Writings of Charles S. Peirce (henceforth 'W' followed by volume number; in bibliography as Peirce (1982-Present)). See W2, 282-284.

${ }^{11}$ For an excellent analysis of Peirce's article and diagram see Campbell 2017, chapter 2.

${ }^{12}$ Incidentally, the journal had been just founded by the very Sylvester whose chemical-algebraic graphs I discussed above, and who was Peirce's colleague at Johns Hopkins University. Indeed, Peirce's own use of the term 'graph' in logic is deeply indebted to Sylvester. In the 'Prolegomena to an Apology of Pragmaticism' he explicitly states: 'By a graph (a word overworked of late years), I, for my part, following my friends [William Kingdon] Clifford and Sylvester, the introducers of the term, understand in general a diagram composed principally of spots and of lines connecting certain of the spots' (CP 4.535). Peirce's complaint that the term was becoming 'overworked' signals that by 1906 it was stretching beyond its logical/algebraic use and coming to be associated with graphical representations such as the ones by Helmholtz and Whewell I discussed in the previous section.

${ }^{13} \mathrm{My}$ aim in what follows is not to give a detailed and technical account of Peirce's diagrammatic system of Existential Graphs, on which there is a constantly growing literature. Instead, I hope to flesh out, from Peirce's philosophical considerations, what his system has to offer to the broader historical and philosophical literature on diagrammatic representations. Technical studies of Peirce's Existential Graphs began in the 1960s and 1970s with the works of J. Jay Zeman (1964) and Don D. Roberts (1974), and saw a revival from the late 1990s: see for example Barwise and Etchemendy, 1996; Shin 2002; Pietarinen 2006; Stjernfelt 2007; Legg 2011; Legg 2013; Pietarinen and Bellucci 2016a and the forthcoming vol. 1 of the comprehensive edition of Peirce's logical writings (Pietarinen, forthcoming).
} 
"You will perceive, however, that the atomic
weights seem to arrange themselves on the diagram in weights seem to arrange themselves on the diagram in
parallel shelving lines. Also, that there is a corres!ondence between the series of art ads and perissads which have the highest atomic weight-that is to say, $\mathrm{Na}$, $\mathrm{K}, \mathrm{Rb}, \mathrm{Cs}$, Tl, on the one hand, and $\mathrm{Mg}, \mathrm{Ca}, \mathrm{Sr}, \mathrm{Ba}$
$\mathrm{Pb}$, on the other, inasmuch as they form strong bases and peroxides, but no suboxiles or acids. A corre-
snondenct is also to be traced between the two series having the next highest atomic weights; that is to say, $\mathrm{Fl}, \mathrm{Cl}, \mathrm{Br}, \mathrm{I}$, and $\mathrm{O}, \mathrm{S}$, Se, Te, innsuuch as they have a strong tendency to unite in simple proportions with ing definite and distinct compounds, and slso form

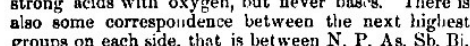
and $\mathrm{C}, \mathrm{Si}, \mathrm{Ti}, \mathrm{Zr}, \mathrm{Sn}, \mathrm{Pt}$, insmuch na they form acids which unite in the most conplicated proportions with bases, putting types at dehince, and also (as far as the higher members of each Beries are concerned) have some tendency to form weak bisc.

"I also notice that there is a much greater difference in chemical clinracters between $\mathrm{K}$ and $\mathrm{Na}$ (not isotnorinsoluble), between $\mathrm{N}$ and $\mathrm{P}$ ( $(\mathrm{N}, \mathrm{O}$ 。 being monobasic), between $\mathrm{O}$ and $\mathrm{S}$, between $\mathrm{C}$ and $\mathrm{Si}$, and between $\mathrm{Gl}$ and $\mathrm{Mg}$, than between any other Iwo aijacent metmbers of their respective series, Albo that the maximum of
resemblance is between $\mathrm{As}$ and $\mathrm{Sb}, \mathrm{Br}$ and $\mathrm{I}, \mathrm{Rb}$ and
$\mathrm{C}_{\mathrm{s}} \mathrm{Sr}: \mathrm{sd} \mathrm{Ba}$, Se and $\mathrm{Te}, \mathrm{Zn}$ and $\mathrm{Cd}$.

"Finally, there $i^{2}$, as you point out, specinl resemblance between elements occunying corresponding places in the series, as between $\mathrm{C}$ and $\mathrm{B}, \mathrm{O}$ and $\mathrm{FL}, \mathrm{S}$ and $\mathrm{P}, \mathrm{Ca}$ and $\mathrm{K}$, $\mathrm{Se}$ and $\mathrm{As}, \mathrm{sr}$, $\mathrm{Sr}$ and $\mathrm{Rb}, \mathrm{Ag}$ and $\mathrm{Pa}, \mathrm{Au}$ and $\mathrm{Os}$, Tl and Pb. \&c."
The table sent to us was drawn on engineers' profile paper, and is ro:newhat easier to be understood and to be verified than the engraving. The engraving is, however, a faithful copy of the original, excepting the
ruled lines. The profile paper and the cross-section ruled lines. The protile paper and the cross-section paper of the engineers is exceedingly convenient for
preparing similar talles and charts. preparing similar tahles and charts.
Our correspondent's table, besides being a rery admirable exposition of the facts of pairiny at the sime time illustrates almost everything of ralue which has been written on the classification of the elementz and the numerical relations of the abmic weights. It will well repay a caref

which we suggest a different number of the elements. Is not our : the prosificable present-of perisssus and artiads, puired groups and paired individuals - about as perlect and satisfactory as

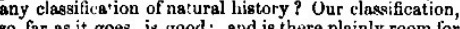
go far as it goes, is good; and is there plainly room for gested that it might be sgreed that some of the unpaired elements are enlowe li with hoth alomicities, and thus be paired with themselves. The suggestion (by no means a new one) is applicable especisly to iron, manganese, ehromium, copper, and mercury. Iron,
in $\mathrm{FeS}$, is an artiad; in $\mathrm{Fe}_{2} \mathrm{O}_{1}$, may it not be $\mathrm{B}$ perissad? Dr. Odling says, "The ferrous and furric atoms have distinct chemical propertics, and form distinct series of compounds, which differ more from one it be deternined that the pairing is an order of nature, that the known groups appear filled, we ought not to ance that had we been unawn groups appear

Fig. 2. Peirce's arrangement of the chemical elements. Peirce (1869), 390. Courtesy of Hathi Trust. Available at:

https://babel.hathitrust.org/cgi/pt?id=mdp.39015073215785\&view=2up\&seq=356 (last visited 30 December 2019). 


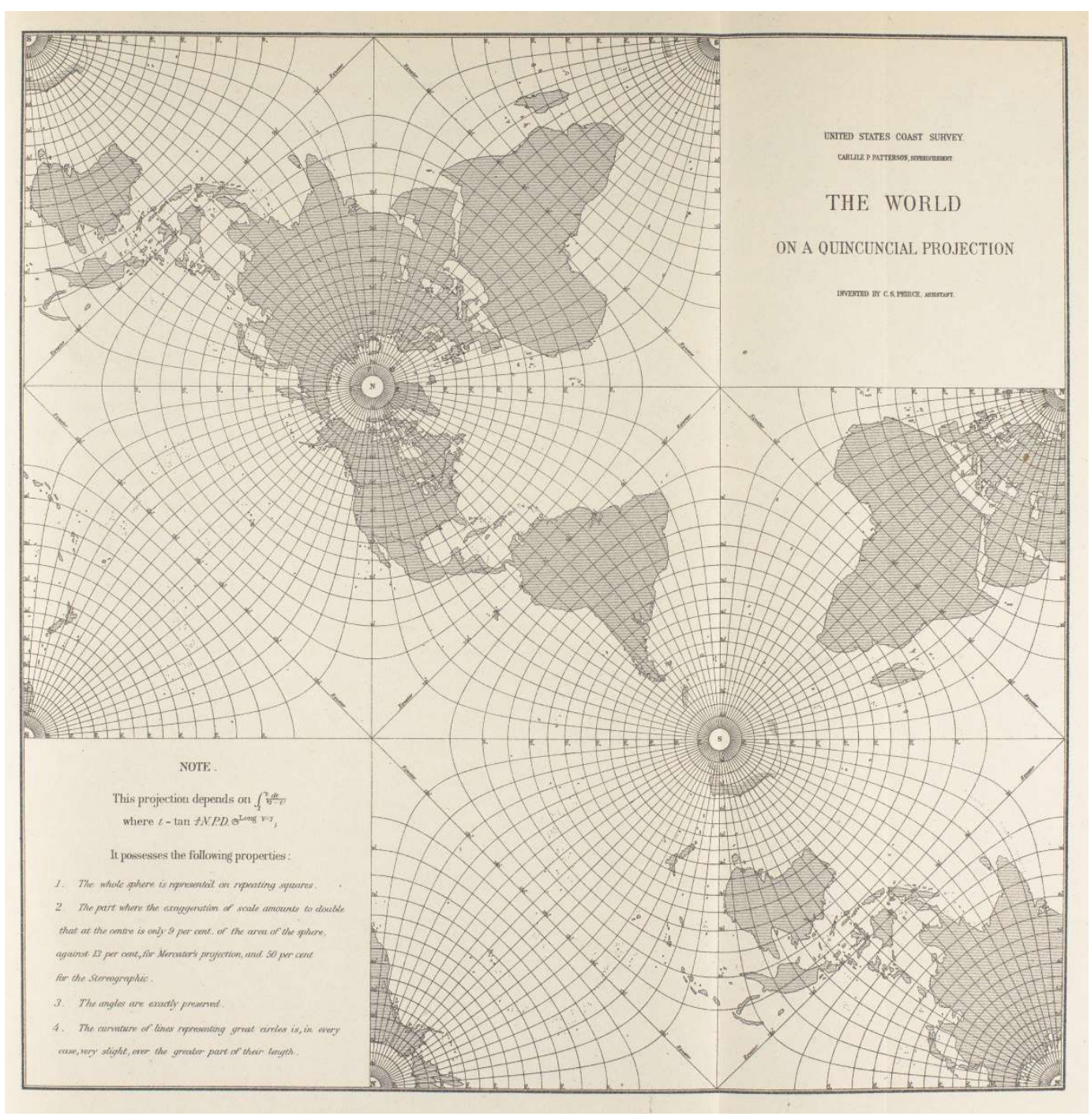

Fig. 3. Peirce's Quincuncial map. Peirce (1879), 397. Courtesy of UCL Special Collections. 


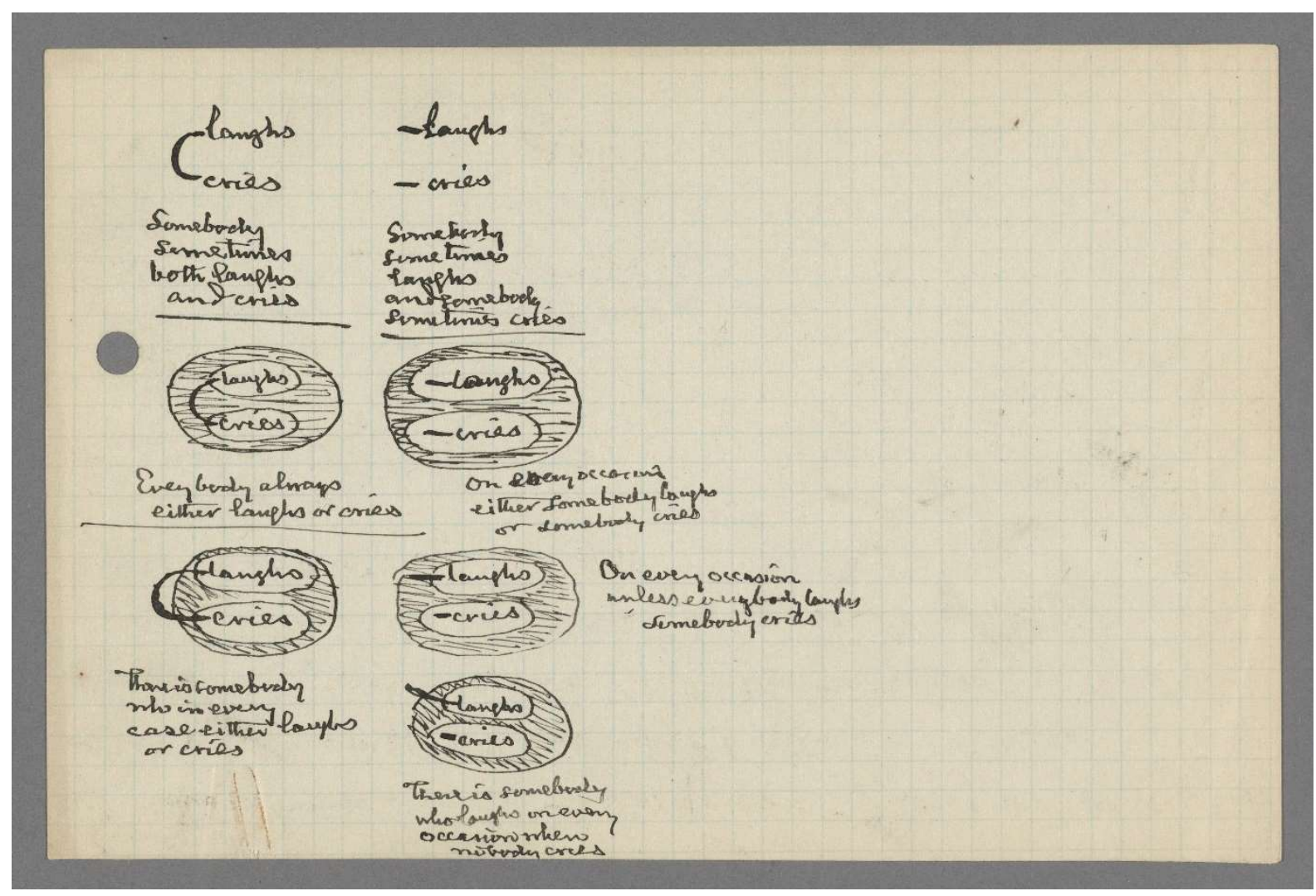

Fig. 4. Peirce's Existential Graphs. R 292 (1906), Prolegomena to An Apology of Pragmaticism, unnumbered sheet. Courtesy of the Houghton Library, Harvard University.

As Ahti Pietarinen $(2006,109 \mathrm{ff})$ notes, the system of Existential Graphs was rooted in Peirce's previous investigations in logical algebra. It is possible that the original motivation for the Existential Graphs dated as far back as 1882, and represented an effort on Peirce's part to improve on the perspicuity and readability of his logic of relatives. Indeed, in a letter to his student and then colleague at Johns Hopkins Oscar Mitchell, Peirce pointed out that his 'notation of the logic of relatives can be somewhat simplified by spreading the formulae in two dimensions' ( $R$ L294, 1882, cited in Pietarinen 2006, 109). Peirce's aim was to build a system of logic that could capture the closest correspondence with the process of reasoning and exhibit this correspondence in the clearest and most perspicuous manner. The diagrams, in Peirce's own words, should function as 'moving pictures of thought' (CP 4.8, c1905; Pietarinen 2006), and in this respect they offered an alternative to dominant approaches to logic in Peirce's time. Indeed, in a later manuscript (R 514, 1909), Peirce explicitly contrasted his own logical system to the drive toward symbolic formalism pursued by his contemporaries:

'So I will break off that and just give an illustration or two of how this Syntax of Existential Graphs works. But before doing that I wish to draw your attention, in the most emphatic way possible, to the purpose this Syntax is intended to subserve: since anybody who did not pay attention to that statement would be all but sure, not merely to mistake the intention of this syntax, but to think that intention as contrary to what is as well he could. Namely he would suppose the object was to reach the conclusion from given premises with the utmost facility and speed, while the real purpose is to dissect the reasoning into the greatest possible number of distinct steps and so to force attention to every requisite of the reasoning. The 
supposed purpose would be of little consequence, and it is the fussiness of the mathematicians to furnish inventions to attain it; but the real purpose is to supply a real and crying need, although logicians are so stupid as not to recognize it and to put obstacles in the way of meeting it' (R 514, 20-21, emphasis added). ${ }^{14}$

The primary requirement of a good system of logic is to supply a 'real and crying need' to force attention to every requisite of reasoning. In the passage, Peirce complains that logicians in his time are placing obstacles to the development of a transparent inquiry into the process of reasoning, by privileging facility and speed over perspicuity and clarity. On the contrary, logic should provide the clearest and most perspicuous way of dissecting reasoning in each of its steps. It is this requirement that his Existential Graphs were supposed to fulfil.

An even more forceful - albeit also more dense - formulation of the perspicuous nature of diagrams is outlined by Peirce in a manuscript ( $R$ 293, c1906) which is probably a draft for what would become his 'Prolegomena to An Apology for Pragmaticism' (CP 4.530ff, c. 1906). A diagram here is defined as 'an Icon of a set of rationally related objects'. Peirce's definition here points to a semiotic category - iconicity - which has become a standard point of reference in the literature on diagrams. Icons, or more precisely iconic representations (as for Peirce an icon is never experienced and used in its pure form) exhibit qualities of the objects they stand for, qualities they would possess 'whether any such Object existed or not' (EP2, 291). ${ }^{15}$ This characterisation of the iconic nature of diagrams is well captured by the short imaginary dialogue I presented in the opening of this section: it is by reasoning on the map's significant parts (which the map would possess, whether its objects existed or not) that Peirce's imaginary General can anticipate novel possible configurations of the army in the battlefield.

But the definition of diagrams as icons of 'rationally related objects' goes even further than this. By 'rationally related' Peirce means 'that there is between them, not merely one of those relations which we know by experience, but know not how to comprehend, but one of those relations which anybody who reasons must have an inward acquaintance with' (R 293, 10-11, emphasis added). This is in line with Peirce's dictum that all necessary reasoning is ultimately diagrammatic ( 293,6$)$, and in particular with his idea that necessary reasoning makes (or should make) its conclusions evident. Reasoning upon a diagram, Peirce explains, allows for the truth of the conclusion to be 'perceived, in all its generality; and in the generality the how and why of the truth is perceived' (R 293, 11, emphasis in the text). His claim here is that iconic representations such as diagrams can provide just this kind of evidence:

'It is, therefore, a very extraordinary feature of diagrams that they show...that a consequence does follow, and more marvellous yet, that it would follow under all varieties of circumstances accompanying the premises'. ( $R$ 293, 13, emphasis in the text) $)^{16}$

\footnotetext{
${ }^{14}$ See John Sowa's detailed commentary on R514, available online at http://www.jfsowa.com/peirce/ms514.htm (last visited 30 December 2019).

${ }^{15}$ This has often being interpreted as Peirce claiming that a relation of resemblance governs iconic representations. I have joined the host of Peirce scholars that have questioned the superficial account of resemblance implicit in these criticisms in Ambrosio (2014). See also Dipert 1996; Hookway 2000, ch.3; Stjernfelt 2000; Stjernfelt 2007; Stjernfelt 2011, and Legg 2011 and Legg 2013 among the most notable defenders of iconicity. The abbreviation 'EP' in the citation refers to the Essential Peirce, edited by the Peirce Edition Project, in bibliography as Peirce (1992-98).

${ }^{16}$ In a brilliant technical article, Cathy Legg (2011) shows that the notion of iconicity involved in Peirce's Existential Graphs should prompt us to revisit the very foundational question of how we come to know
} 
Two considerations are in order here, before moving on to a discussion of how the rediscovery of Peirce's Existential Graphs has defined and shaped current debates on diagrammatic representations. First, a point about perspicuity and communicability. Diagrams for Peirce are (among other things) an opportunity to reflect on the evidential status of logical relations. On the one hand, he claims, relations are discovered through the very process of constructing and inspecting a diagram. This process requires the diagram to be built in relation to an Interpretant which in Peirce's (not always straightforward) terminology is the interpreting sign that a sign itself triggers in an interpreter's mind (or quasi-mind). Once a diagram has incorporated an intention to appeal to an Interpretant, its necessary conclusions become communicable among the members of a community, and thus evident - that is, appealing to 'those relations which anybody who reasons must have an inward acquaintance with' (MS 293, 10-11). Here I use 'evident' in a way that is somewhat analogous to Whewell's considerations about the Method of Curves. While Whewell and Peirce's graphical systems are very different in aims and applications, their motivations for pursuing a diagrammatic method share an analogous sentiment: just like graphical methods make ideas public, so Peirce's graphs trace the movement of thought in reasoning in a way that renders reasoning amenable to the scrutiny of a community.

Secondly, in a time of debates in philosophy of logic and mathematics around the epistemological status of visual proof, Peirce defended the mixed nature of reasoning, showing that such debates missed the mark: each side of the divide between images and symbolic formalisations only captured part of what reasoning amounts to. A property of diagrams is that they work as a special combination of all three kind of semiotic categories that Peirce saw as distinguishing the relationship between a sign and its object. Along with being predominantly iconic (which, as we just saw, Peirce did not exclusively identify with narrowly 'visual'), diagrams obey particular conventions which constrain the range of admissible operations upon them, and function with the indispensable aid of indices as pointers for relevant features to be manipulated and experimented upon:

'A Diagram is a representamen [Peirce's later term for a sign-vehicle or signifying item] which is predominantly an icon of relations and is aided to be so by conventions. Indices are also more or less used. It should be carried out upon a perfectly consistent system of representation, one founded upon a simple and easily intelligible idea' $(R 492,1)$

These additional features delimit and stabilise the 'significant' parts of the diagram, at the same time evidencing that the key to diagrammatic representations is in their heterogeneous nature.

The rediscovery of this particular aspect of Peirce's logical system marked a turning point in the resurgence of diagrams in logic and epistemology in the second half of the twentieth century. In the 1990s, Jon Barwise, John Etchemendy and their students drew directly on Peirce to develop an approach to diagrams which crucially revolved around a view of reasoning and proof as heterogeneous and multi-modal. The starting point of Barwise and Etchemendy's (1995; 1996) programme is a systematic defence of the legitimacy of visual representations as non-superfluous in reasoning and integral to sound and valid proof. Valid deductive inference, they argue, amounts to the reliable extraction of information that is implicit in information already obtained. Language is only one of the ways in which information can be couched, and there is no reason why valid deductive inferences should be modelled exclusively as relations between sentences: a great deal of

mathematical necessity, for which she provides a phenomenological argument grounded in 'the hardness of the iconic must'. Albeit Legg does not cite this specific passage, I believe her account illuminates precisely (among other things) the sense of 'necessity' here invoked by Peirce. See Legg 2011. 
valid reasoning takes place through graphs, diagrams, maps, as well as other media (Barwise and Etchemendy 1996, 4). Sun-Joo Shin (2002) provides a clear overview of the main tenets of this programme:

'Our ordinary reasoning typically involves information obtained through more than one medium - sentences, diagrams, smells, sounds and so on. Recognising the actual practice of this multi-modal reasoning, researchers have started focusing on multi-modal, or heterogeneous representation systems, which employ both symbolic and diagrammatic elements. This is a clear departure from the major direction taken by logicians and mathematicians since the development of modern logic: for more than a century, symbolic representation systems have been the exclusive subject of formal logic' (Shin 2002, 1).

In order to substantiate this claim, the defenders of the validity of multi-modal reasoning have embarked on the ambitious task of proving that diagrammatic systems can be sound and complete just like symbolic systems. Barwise and Etchemendy's views converged into Hyperproof, the first computer programme which combined first-order logic and diagrams to teach elementary logic (Barwise and Etchemendy 1994). Shin's (1994; 2002) groundbreaking work on Venn diagrams and on Peirce's Existential Graphs proved that both systems are sound and complete, once clear syntactical rules are defined to control their use. A major contribution of this programme in logic has been the conclusion that 'there is no principled distinction between inference formalisms that use text and those that use diagrams. One can have rigorous, logically sound (and complete) formal systems based on diagrams'. (Barwise \& Etchemendy 1995: 214).

While revolutionary in its rehabilitation of diagrammatic reasoning, this programme has raised some important criticisms. Mancosu (2005) highlights that there are at least two ways of interpreting its conclusions. If the claim advanced by proponents of multi-modal reasoning is that there are forms of valid visual or diagrammatic reasoning which cannot be expressed in linguistic form, then it is hard to see how this body of works proves the point. 'Indeed' Mancosu states, 'even setting up the question in such a way is problematic, for there is very little clarity on what criteria one can appeal to in order to distinguish linguistic systems from visual systems' (Mancosu 2005, 25). However, far more promising is the way in which these accounts tackle the foundational issue of reasoning with diagrams, showing successfully that visual systems are not inherently deceptive - or at least no more than linguistic systems might be.

In an article that will turn out to be crucial to the rest of my discussion, Valeria Giardino (2013) takes this line of criticism a step further, at the same time using it as an opportunity to build a positive account of diagrammatic representations in philosophy of science. She importantly points out that proponents of multi-modal reasoning remain anchored at once to a view of proof as the centrepiece of mathematical and logical knowledge, and - Shin's (2002) programmatically broad definition of multimodal reasoning notwithstanding - to a bias toward the visual appearance of diagrams as their defining characteristic. Hence, in order to participate in rigorous proofs, diagrams qua visual representations need to be 'tamed' (Giardino 2013,136) by recourse to explicit syntactic rules that fix their meanings. Along with maintaining the old distinction between discovery and justification (with proof falling squarely in the latter camp), this approach seems to imply that all there is to diagrams is indeed their visual appearance, combined with the presence of clearly specified syntactic rules that dissipate the potential ambiguity inherent in their visual form. But as we saw from Peirce's long quote above, the role of syntax is neither to accelerate reasoning nor to compensate for the inherent ambiguities of visual representations. It is to 'supply a real and crying 
need' to force attention to every requisite of reasoning - to make the discovery of significant relations intersubjectively communicable.

To overcome the strange paradox implicit in accounts of multi-modal reasoning, Giardino (2013) proposes an operational framework which crucially hinges on Peirce, and which resonates with the very passage I presented at the opening of this section. The imaginary General's proposal to use pins on a map to experiment with possible configurations on the battlefield is a distinctively material illustration of the equally material and practical set of manipulation procedures that are indispensable to the construction, interpretation and use of diagrams. In an analogous way, Giardino argues that what defines diagrams is neither their superficial, visual appearance nor the presence of syntactic rules that govern their use. Instead, the operations we can contextually perform on and with diagrams to give structure to a problem are what fixes their meaning: 'Once we are familiar with a specific practice', she argues, 'we manipulate diagrams in meaningful ways, engaging ourselves in a form of reasoning that is stable because it is shared by a community and thus constitutes at the same time discovery and justification for that discovery' (Giardino 2013, p. 138, emphasis added). This definition is semiotic, and more precisely Peircean, in a number of productive ways. At a basic level, diagrams are always produced and interpreted within a context of use. This might seem a trivial point, but it is an indispensable one to show, via the very notions of experimentation and manipulation, the continuity between the (only apparently) abstract fields of mathematics and logic and other areas of scientific practice. And indeed this is a point Giardino herself highlights, in referring to the dynamic nature of Peirce's account of diagrams: 'Diagrammatic reasoning would then bring logic and mathematics closer to the natural sciences: logicians and mathematicians experiment with the very same representations that constitute their instruments' (Giardino 2013, 141). Moreover, in this account diagrams are explicitly representational. Here Giardino adopts once again a Peircean definition of representation, as 'that character of a thing by virtue of which, for the predication of a certain mental effect, it may stand in place of another thing' (Peirce, CP 1.564; Giardino 2013, 141). However, she also points out that this formulation should not be taken too literally: diagrams are representational not because they depict or correspond to some pre-existing abstract object, but because they dynamically embody relevant relations that are interpreted contextually as such by a community of practitioners, and that can be discovered and shared through manipulation. Thus manipulation is the bridge between the public dimension of diagrams and the argument that it is their heterogeneous nature that counts: we manipulate ensembles of visual and symbolic/linguistic elements, and it is neither the exclusively visual nor the exclusively formal nature of diagrams that makes a real difference. It is how we use diagrams to structure problems and discover and stabilise relations that makes them a particularly fruitful kind of representation.

It may be argued that Giardino's account is conflating 'representational' with 'relational', ${ }^{17}$ and to a certain extent her phrasing might lend itself to this objection. To understand the subtlety of her point it is worthwhile returning to Peirce, and in particular to the opening pages of the 'Prolegomena to an Apology of Pragmaticism'. Immediately after drawing the comparison between chemistry as a way of putting questions to nature and diagrams as a way of putting questions 'to the Nature of the Relations involved'(CP 4.530), Peirce pre-empts a possible criticism which clarifies, among other things, a sense in which logical diagrams may be considered 'representational'. One might argue, Peirce acknowledges, that there may be 'a good deal of difference between experiments like the chemist's, which are trials made upon the very substance whose behaviour is in

\footnotetext{
${ }^{17}$ I am grateful to Harro Maas for alerting me to this possible objection.
} 
question, and experiments made upon diagrams, these latter having no physical connection with the things they represent' (CP 4.530). But this narrowly referential sense of 'representational' misses the point, in that it assumes that chemists merely manipulate individual samples 'which could very well be thrown away, as having no further interest' beyond the experiment itself (CP 4.530). Instead, Peirce claims, the chemist's quest is a quest for generality: 'for it was not the particular sample that the chemist was investigating', Peirce reiterates, 'it was the molecular structure' (CP 4.530). As Christopher Hookway (2012) has observed, in this case the chemical sample serves as an iconic representation of any other sample of a substance, precisely because it shares with them the same molecular structure. Thus molecular structure is the object of chemical experimentation, 'and since it is present in every sample, the researcher experiments upon the 'Very Object under investigation' every time that she experiments upon a particular sample' (Hookway 2012, 119). In an analogous way, the object of diagrammatic reasoning is neither a particular nor an abstract object, but 'the form of a relation'. Peirce continues with a mathematical example:

'Now this Form of Relation is the very form of the relation between the two corresponding parts of the diagram. For example, let $\mathrm{f} 1$ and $\mathrm{f} 2$ be the two distances of the two foci of a lens from the lens. Then,

$1 / \mathrm{f} 1+1 / \mathrm{f} 2=1 / \mathrm{f} 0$

This equation is a diagram of the form of the relation between the two focal distances and the principal focal distance; and the conventions of algebra (and all diagrams, nay all pictures, depend upon conventions) in conjunction with the writing of the equation, establish a relation between the very letters $\mathrm{f1}, \mathrm{f2}$, f0 regardless of their significance, the form of which relation is the Very Same as the form of the relation between the three focal distances that these letters denote' (CP 4.530).

Two points here are worthwhile noting. First, just like different samples of a substance share the same molecular structure, so in Peirce's mathematical example the object of the diagrammatic representation - in this case an equation - is the form of the relation between the three focal distances, independently of the contingent features of its material notation. It is in this sense that both the chemist and the mathematician/logician experiment upon 'the Very Object under investigation' (CP 4.530). Moreover, and perhaps more importantly, here Peirce is suggesting that an equation can function as a diagrammatic representation precisely insofar as it makes evident, through shared conventions and through the act of writing of the equation, the form of such a relation between the three focal distances.

What Giardino's account of the representational nature of diagrams captures from Peirce is a broad and dynamic definition of representation: diagrams qua representations are not static correspondences with pre-existing objects. Instead, as Giardino argues, it is through the process of constructing diagrammatic representations and manipulating them that mathematicians and logicians discover and stabilise relations (logical relations, relations between mathematical quantities, etc.), and use them to structure particular problems. Moreover, the representational nature of diagrams, and the set of shared manipulation practices associated with them, is also what renders the 'form of a relation' evident and communicable within a community of practitioners: it would be hard to explain the public nature of diagrams without appealing to their (dynamically) representational nature. By standing in place of relevant relations for a community of practitioners/interpreters (to take up again, and fill in, the definition of representation that Giardino borrowed from Peirce), diagrams acquire public significance. 
That this sense of 'representational' may be coextensive with 'relational' is precisely one of the distinctive features of diagrams, at least in a Peircean sense, and it is intimately connected with Peirce's claim that experimenting on a diagram consists of experimenting on 'the Very Object' of investigation - thus bringing logic closer to experimentation in the natural sciences. With the exception of Giardino's operational account, this particular aspect of Peirce's approach to diagrams has remained underexplored in the literature, which has instead privileged either the notion of iconicity in diagrammatic representation, or the role of Peirce's Existential Graphs in defending more broadly the validity of multi-modal reasoning. And yet, the idea that experimenting with a diagram is a way of coming into contact with the object of inquiry that the diagram is supposed to investigate, and that this sense of 'experimenting' is not at odds with a diagram's representational nature, can serve as a springboard to overcome a broader tension lurking in the background of current historical and philosophical approaches to diagrams in the literature. In the final section of this critical review I present this tension through the (intentionally artificial) labels of 'the object-based view' and the 'representational view' of diagrams. I sketch a possible explanation for this divide in the literature in dialogue with the historiographical analyses of the 'eclipse of diagrams' I presented in the first part of this review, and conclude by offering a proposal for reconciliation.

\section{The Object-Based View versus the Representational View of Diagrams: a False Dichotomy?}

In a brief reflection included in the final section of the collection Representation in Scientific Practice Revisited (2014), Lorraine Daston invites scholars in Science and Technology Studies to 'go beyond' the epistemological notion of representation, calling for a shift from the epistemology to the ontology of images. 'What would an ontological account of scientific images look like?' Daston asks. 'For starters it would collapse the distance between presentation and representation: the image is the presentation, the working object of science' (Daston 2014, 320-21). Daston here has in mind contemporary computational imaging techniques such as computer simulations, but her invitation to move beyond representation is symptomatic of a broader discomfort toward philosophical accounts of representation that have privileged 'what we know' at the expenses of how practices of image-making have provided the sciences with their working objects (Daston 2014, 320-21).

A similar tension between epistemology and ontology seems to lurk in the background of the literature on diagrams. Philosophical accounts, as I showed in the course of my discussion, take the representational nature of diagrams as an important point of departure for further investigation. But this is by no means the only view on the matter, and historians as well as scholars in the field of visual culture have explicitly distanced themselves from invoking the concept of representation in relation to diagrams. Thus, for example, in The Culture of Diagrams (2010), John Bender and Michael Marrinan state that diagrams are 'closer to being things than to being representations of things' (Bender and Marrinan 2010, 21). In a recent special issue of Endeavour on diagrams as 'tools of reason', Greg Priest, Silvia De Toffoli and Paula Findlen follow this characterisation, stating their preference for 'consider[ing] diagrams as objects that exist in the world' rather than 'visual representations of aspects of the world' (Priest, De Toffoli and Findlen 2018, 52). ${ }^{18}$ In what follows, I

\footnotetext{
18 Interestingly, the special issue also features an article by Giardino (2018), which argues - among other things - that the view of diagrams as tools of reason should not be considered at odds with their representational nature.
} 
will subsume these two (broad) families of approaches under the labels of 'the representational view' and the 'object-based view' of diagrams.

Partly inspired by Wise' (2006) plea for intersections in the analysis of practices of 'making visible', I want to draw from the literature the very bases to challenge this dichotomy. This is also an opportunity to probe the position that diagrams occupy within the broader, and now fully consolidated, field of 'visual culture', especially in its recent connections with the history and philosophy of science. In a recent paper, Ahti Pietarinen and Francesco Bellucci (2016a, 174-175) hold the current interest in 'visual culture' and 'visuality' responsible for the widespread view that diagrams are visual in a sense that symbolic notations are not - a tendency which they describe as one of the 'dogmas of diagrammatic reasoning'. In their argument they take a hard logical line: 'the visual character [of diagrams]', they state, 'gains prominence only as an accidental characteristic of thought. Human characteristics are hardly the hallmarks of what real reasoning consists of' (Pietarinen and Bellucci 2016, 175, emphasis in the text). Of course, there are a range of compelling reasons to reject accounts of diagrams that prioritise their primarily visual (as opposed to symbolic) nature - and indeed the operational view proposed by Giardino which I outlined in the previous section is an excellent example of how this can be done. However, the way in which Pietarinen and Bellucci present their objection is a classic example of why historians and scholars in Science and Technology Studies interested in visual culture might have wanted to distance themselves from the set of philosophical attitudes and assumptions that Daston (2014) subsumes under the broader category of 'epistemology'. For one thing, visual culture is precisely one of the fields through which the study of diagrams has been thriving - as a material, socially located, and historically contingent practice. ${ }^{19}$ Thus, in the final part of this review, I will attempt to flesh out from the literature an important sense in which the 'visual' in these accounts might include practices of manipulability and intervention that take us beyond establishing 'new dogmas' of diagrammatic reasoning.

The locus classicus for the beginning of a new historiography of visual methods in the sciences is Martin Rudwick's (1976) 'The Emergence of Visual Languages in Geology, 1760-1840', now recognised as a turning point in the systematic study of the visual dimension of science in its various manifestations.$^{20}$ There, Rudwick inscribes topological maps and diagrams within the broader array of visual resources that were integral to the emergent discipline of geology. He points out that the neglect of visual displays and visual practices was partly a result of the history of the history of science itself, whose practitioners were trained in a tradition that privileged 'numeracy' and 'literacy' - mathematical formalism and language - as primary modes in the production and transmission of scientific knowledge. Crucially, and with a nod to the logical positivist tradition I briefly sketched at the beginning of this review, Rudwick complains that philosophers of science have not been particularly alert to the visual dimension of science either: 'The philosophy of science, with which the history of science has had institutional links of great distinction, represents as it were an amalgam of both 'numerate' and 'literate' traditions' (Rudwick 1976, 150).

In another defining historical study, David Kaiser (2005) motivates his emphasis on the role of tacit knowledge and the larger pedagogical context surrounding the 'dispersion' of Feynman diagrams through a contrast with the theoretical approaches pursued by philosophers of science: 'in these traditional [philosophical] accounts, the skilled manipulation of tools played little role: theorists were assumed to write papers whose content other theorists could understand, at least in

\footnotetext{
${ }^{19}$ A landmark study in this respect is Elkins 1999.

${ }^{20}$ Rudwick's article had a large influence on the history of science and its methodology. For a recent review of its significance in the field, see Kusukawa 2016.
} 
principle, anywhere in the world' (Kaiser 2005, 8). Thus philosophy is seen to perpetuate a view of language and formalisation as the royal roads to universal communicability, in a sense analogous to the one described by Daston and Galison as 'structural objectivity'. To this received view, Kaiser presents a counternarrative in which Feyman diagrams are calculational tools, whose functioning is explicitly in contrast to the 'philosopher's dream world': 'theorists have used calculational tools... to mediate between various kinds of representations of the natural world. These tools have provided the currency of everyday work' (Kaiser 2005, 8-9). ${ }^{21}$

There are two senses in which criticisms of the kind expressed in Rudwick's and Kaiser's studies might have informed the 'object-based view' of diagrams. One relates to a general distrust for a reputation philosophy built for itself, and which philosophers still struggle to shake off (when they are willing to confront it in the first instance). The foundational debates in mathematics and logic I outlined in the first part of this review, and especially their effects on the shape and history of analytical philosophy, are an example of how philosophers came to gain such a reputation. The 'visual' in this case becomes distinctive of a disciplinary identity and historiographical stance construed explicitly in contrast with this kind of philosophical agenda (or contemporary vestiges of it), and is a surrogate for the far broader range of material, historically contingent practices that could not be accounted for by strict formalisms, empirically verifiable hypotheses, and rigorous proof.

The other sense is closely connected to the recent attention to representations in philosophy of science, an area in which diagrams are progressively gaining visibility. ${ }^{22}$ In an important philosophical contribution to this debate, Mauricio Suárez (2010) has shown that the current literature on scientific representation is divided along two distinct conceptual axes. On one hand, an 'analytical' axis of inquiry draws together philosophical accounts that use the concept of representation to investigate the relation between theory and the world. These accounts concern themselves mainly with problems of meaning, reference and the metaphysics of relations. They cast representation as a relation between a source (the vehicle of the representation) and a target (the object of the representation), which are considered as mere placeholders. The aim of these accounts is to provide an analysis of the formal relation holding between source and target - in other words

\footnotetext{
${ }^{21}$ There is much more to Kaiser's account than the brief sketch I provide here. The very title of his book on Feynman diagrams, Drawing Theories Apart, is an explicit rejoinder to Bruno Latour's 1990 'Drawing Things Together': 'The very idea of following a nonhuman scientific object around as an organising principle bears a certain Latourian signature. Yet, I follow a different line when it comes to the question of 'immutable mobiles'...Whereas Latour emphasizes 'optical consistency' (even 'immutability') as an essential feature of why diagrams and other scientific inscriptions carry so much force among scientists, I focus instead on unfolding variations within their work - on the production and magnification of local differences and the work required to transcend those differences' (Kaiser 2005, 7).

${ }^{22}$ See for example Meynell 2008, who provides an answer in terms of representations to the claim that Feynman diagrams are calculational devices; Meynell 2018 takes up again the discussion about the representational nature of Feynman diagrams and relates it to a non-factive account of understanding. Perini 2013 and Perini 2010 discuss diagrams and representations in biology; William Bechtel, Adele Abrahamsen Daniel Burnston and Ben Sheredos have worked for a long time now on representing biological mechanisms through diagrams - see for example Sheredos et al 2013. On mechanism diagrams in cell biology, particularly apoptosis research, see $\mathrm{Au}, 2016$. On diagrams in relation to models and representations in economics see Morgan 2012, esp. ch 3. Giardino 2013 and 2018 place mathematical diagrams in dialogue with debates on representation in philosophy of science. Ambrosio 2014 contains my own contribution, via Peirce, to this expanding literature.
} 
they focus on what Suárez defines as the constituents of representation. ${ }^{23}$ The 'practical inquiry', on the other hand, exemplifies the contemporary tendency to integrate history, philosophy and scientific practice in a unified account. Rather than focusing on what constitutes the relation of representation, these accounts concern themselves particularly with the practice of model-building and with the ways in which scientists actually use models. Their starting assumption is that the relation of representation cannot be captured by appealing to mathematical or formal structures, and that the debate around representation should be shifted to a concrete investigation of the various uses of models and the particular contexts in which they are used. ${ }^{24}$

While helpful in charting the field, this distinction has also contributed to feed further dichotomies, the most worrying one being that an interest in the question of what constitutes representation should exclude scientific practices in their contingent, historical unfolding - and vice versa, that an attention to history should come at the expenses of an inquiry into what constitutes a representation in the first place. It is perhaps a caricatured version of the representational view of diagrams as belonging purely to a quest into an 'analytical' type of inquiry that the object-based view aims to avoid, by renouncing to the representational aspects of diagrams altogether. And it is this same caricatured version of representation that motivates its conflation with 'epistemology', as expressed by Daston (2014) in her plea to a shift to the ontology of working objects instead.

But as I started suggesting through the case of Peirce in the previous section, a view of diagrams as objects of inquiry in their own right and a view of diagrams as representations need not be considered as mutually exclusive - provided we extend the notion of representation beyond a mere 'analytical' relation between a source and a target. This extended view of representation has been recognised, and defended, by scholars in the field of Science and Technology Studies in a number of ways. For example, in response to Daston's plea to move beyond representation, David Lynch (2014) has highlighted how abandoning the concept altogether 'would not unload the baggage' it carries. More productively, he suggests to revisit it with an emphasis on the range of practices involved in representing, to 'draw attention to the many ways in which words do more and other than refer, pictures do more and other than depict, and representations do more and other than correspond to objects and/or ideas' (Lynch 2014, 325). In a similar vein, Steve Woolgar (2014), himself a proponent of the 'ontological turn' in Science and Technology Studies (Woolgar and Lezaun 2013), acknowledges that there may be some benefits in cultivating, rather than severing, the links with representation. For one thing, representation is hard to avoid: 'As with the rock star trying to reinvent himself', Woolgar argues with a colourful metaphor, 'something of his identity stubbornly

\footnotetext{
${ }^{23}$ This relation is usually construed as a similarity or (partial) isomorphism between a source and a target. Defenders of similarity (and selective similarity) comprise, among others, Giere (1988), Giere (2004), the early Van Fraassen (1980), van Fraassen (2008) and Teller (2001). On the isomorphism (or partial isomorphism) side of the debate are Da Costa and French (2003). Van Fraassen's (2008) 'structural empiricism' hinges on a notion of selective similarity that ultimately is explained in terms of (structural) isomorphism, whereas Giere (2004) proposes a much weaker notion of similarity that refrains to appeal to 'structure'.

${ }^{24}$ Practice-based accounts are a result of the most general "turn to practice" that has characterised philosophy of science starting from the 1980s. The forerunners of this approach are Achinstein (1968), Black (1962) and Hesse (1963). Cartwright (1983), Hacking (1983) and Morrison and Morgan (1999) pioneered the shift toward practice-based approaches to models; Gooding (1990) importantly challenged the sharp divide between observing, experimenting and representing, whereas De Chadarevian and Hopwood (2004) examined the historical context surrounding the production and use of models. Useful overviews of this debate are in Frigg and Hunter (2010) and Suárez (2010). The turn to practice in philosophy of science has a sociological counterpart in the accounts of representation in practice presented in Lynch and Woolgar (1990), and its recent revisited edition by Coopman, Vertesi, Lynch and Woolgar (2014), which features the brief commentary by Daston I presented at the beginning of this section.
} 
remains' (Woolgar 2014, 331). More importantly, a systematic inquiry into the range of practices associated with particular kinds of representations affords us to ask broader questions about their significance, authority and circulation in the scientific community.

Diagrams are representational in this broader sense. Recall here the representational aspect, via Peirce, of Giardino's operational account of diagrams: diagrams represent not because they mirror or correspond to some pre-existing object, but 'because they embody relevant relations' (Giardino 2013, 141), which are contextually interpreted as such, shared amongst practitioners, and actively deployed to structure particular problems. 'When one learns to use a certain diagrammatic system for performing some inferences', Giardino continues, 'she learns a manipulation practice. The diagram becomes the mathematician's worksite, where operations, plans, and experiments are made in order to find solutions and reasons for these solutions'. (Giardino 2013, 145-146). The representational nature of diagrams is thus inextricably related to practices of manipulability; it is what provides diagrams with shared procedures to structure problems and experiment upon their possible solutions.

This sense of manipulability - of doing things with diagrams by exploiting their representational and mediating nature - also helps toning down the unwarranted scepticism toward considering them as part of 'visual culture'. True, the 'visual' nature of some diagrams does not exhaust their properties and uses, and does not necessarily explain how we reason with them. But very few studies in 'visual culture' focus exclusively on the superficial appearance of particular representations to address their role in knowledge-making. Instead, they construe the 'visual' as a compound of practices that extend beyond the pictorial format and incorporate the historical, material and contextual aims and conditions for those practices. Historians of science building bridges with the broader field of visual culture have noticed this. Thus, for example, in his account of Michael Faraday's visual reasoning, David Gooding $(1990 ; 2006)$ appeals to a broader notion of 'construals' as a whole phenomenology of situated cognition, which integrates interpretative images, objects and language with other senses and capacities, and which is importantly continuous with deliberative manipulation. 'Visualisation integrates different types of knowledge and experience' Gooding claims. 'Much of the cognitive power of images resides in this integrative capability, which is central to inference in many sciences' (Gooding 2006, 58). In a similar spirit, but with an emphasis on the role of diagrams in pedagogical traditions and practices, Matthew Eddy (2014) discusses the chemical diagrams produced and used by Joseph Black's students in Edinburgh in the last three decades of the eighteenth century as 'pictures that were composites of visual concepts, materials and practices' (Eddy 2014, 181). And indeed the 'compositional' reading Eddy develops for Black's diagrams is borrowed directly from studies in visual cultures, visual anthropology, and history of art, all stressing the continuity and integration between visual, material, and contextual aspects of knowledge-making. ${ }^{25}$

Dichotomies tell us more about the identities of the practitioners that construct them than about the objects of inquiry they aim to delimit and demarcate. They are, in Wise's $(2006,79)$ perceptive formulation, a product of the 'either/or of intellectual conceit'. The historiography of diagrams and the philosophical debates surrounding their epistemological status are a case in point. The standard story of the 'eclipse of diagrams' in the nineteenth century had the effect of generating a view of philosophical inquiry as explicitly 'anti-visual', theoretical, and formal in the eyes of historians and scholars of visual culture, while at the same time, it strengthened 'the visual versus

\footnotetext{
${ }^{25}$ Eddy cites Baxandall (1972), Alpers (1983), and especially Mitchell (2005), as the pioneers of the compositionalist approach in history of art.
} 
linguistic' dichotomy in philosophical analyses that purported to rehabilitate the epistemological status of visual representations. Similarly, the suspicion toward a narrow - and a times caricatured view of representation as a static correspondence or mere 'mirroring' of a clearly delimited portion of phenomena has led to the dismissal of the representational aspects of diagrams altogether. But in fact the very mixed and dynamic nature of diagrams is the common ground over which all these approaches converge, over which dichotomies blur into intersections, and over which the relative merits of logical, epistemological, historical and contextual accounts of diagrams find important areas of overlap and interaction.

\section{Conclusions}

'Focusing on one or another form of scientific sight keeps two questions front and center: what kinds of practices are needed to produce this kind of image? And what kinds of practices are needed to cultivate the scientific self so that this sight is possible? The history of scientific sight always demands this double motion, toward the unfolding of an epistemology of images, on one side, and toward the cultivated ethics of the scientific self, on the other' (Daston and Galison 2007, 382)

As Daston and Galison remind us, forms of scientific sight, practices of image making and the fashioning of particular kinds of 'scientific self' are inextricably related. The historical line I pursued in this review shows how this claim applies as much to diagrams themselves - as representational practices at the intersections between the formal and the visual - and to the scholars who theorised their roles in knowledge-making.

In this critical - and very partial - overview of the scholarship on diagrams, I have advanced three main points. First, I have attempted to sketch the premises for an alternative historiography of diagrammatic representations, by paying attention to important insights deriving from advocates of diagrammatic reasoning in a time of 'anti-visual puritanism' and 'objectivity without images'. In doing so, I hope to have shown that paying attention to practices at the intersections between numerical and graphical, formal and visual methods is more informative for an understanding of the roles and functions of diagrams in knowledge-making than the partial explanations we might derive from drawing dichotomies. Secondly, through a close look at Peirce's long engagement with diagrams and at the legacy of his semiotic and logical works, I have tried to show that the representational nature of diagrams is crucially intertwined with their public dimension: diagrams for Peirce make relations evident 'to anyone who reasons'. Practices of manipulation and experimentation are the very bridge between the heterogeneous nature of diagrams and their fundamentally public nature: as we saw through Giardino's (2013) operational account, learning to draw inferences from a diagram involves learning the use of a manipulation practice to engage 'in a form of reasoning which is stable because it is shared by the members of a community' (Giardino 2013,138 , emphasis added). Lastly, the heterogeneous and dynamic nature of diagrams is the very ground over which disciplinary dichotomies dissolve, clearing out precious space for interaction and integration. As representations and objects of inquiry in their own right, as visual, formal, material, and historically contingent practices, diagrams inhabit the very intersection where logic, epistemology, history and visual culture in the past century decided to part ways. It is the re-opening of that temporarily interrupted dialogue that I hope to have encouraged, in this critical overview of the history and philosophy of diagrammatic practices. 


\section{Acknowledgments}

This article owes a great deal to a collegial and international community of scholars. I am grateful to Harro Maas for inviting me to write a contribution to this special issue, and for his careful and collegial critical commentary on an early draft of this piece. I benefited immensely from the activities of the 'All-London Integrated HPS' reading group, which took place at the LSE in the spring 2019. I am especially grateful to Mary Morgan, Dominic Berry, Roman Frigg, James Nguyen and the members of the Narrative Science Project for agreeing to run a whole iteration of this annual event on the topic of diagrams, and for the lively and helpful discussion we had in each session. My PhD student Claudia Cristalli read an early draft of this article and provided precious comments, subverting once again in her unique way our supervisor-supervisee relationship. The indefatigable community of Peirce scholars, particularly Cathy Legg, served as my compass in a rather large field and guided my journey through the literature. Steven Wright at UCL Special Collections provided invaluable (and invaluably prompt!) support with the reproduction of the images from the American Journal of Mathematics; I am also grateful to the Houghton Library at Harvard for the reproduction of Peirce's diagrams. Once again, and as always, Niall Le Mage kept track of the word count, commented every section, witnessed me agonise over word choices and image files resolution, and added a whole new list of diagram jokes to our repertoire.

\section{References}

Achinstein, Peter (1968). Concepts of Science. A Philosophical Analysis. Baltimore: Johns Hopkins Press.

Ambrosio, Chiara (2014). 'Iconic Representations and Representative Practices', International Studies in Philosophy of Science, vol. 28 no 3, pp. 255-275.

Ambrosio, Chiara and Campbell, Chris (2016). 'The Chemistry of Relations: Peirce, Perspicuous Representations and Experiments with Diagrams', in Kathleen A. Hull and Richard Kenneth Atkins (eds.) Peirce on Perception and Reasoning: From Icons to Logic. New York and London: Routledge, pp. 86-106.

Au, Yin Chung (2016), Synthesising Heterogeneity. Trends of Visuality in Biological Sciences circa 1970s-2000s. PhD Thesis, University College London. Available electronically at http://discovery.ucl.ac.uk/1478180/13/YCAu Thesis\%202016 copyright\%20materials\%20removed. pdf (last visited 14 August 2019).

Ayer, A.J. (1953). Language, Truth and Logic. London: Dover.

Barwise, Jon and Etchemendy, John (1994). Hyperproof, Stanford: CSLI Publications.

Barwise, Jon and Etchemendy, John (1995). 'Heterogeneous Logic', in J. Glasgow, N. Hari Narayanan, and B. Chandrasekaran, (eds), Diagrammatic Reasoning: Cognitive and Computational Perspectives, pages 209-232. Cambridge, Mass.: AAAI Press/The MIT Press.

Barwise, Jon and Etchemendy, John (1996). 'Visual Information and Valid Reasoning', in Gerard Allwein and Jon Barwise (eds.) Logical Reasoning with Diagrams, New York and Oxford: Oxford University Press, pp. 3-25.

Bender, John and Marrinan, Michael (2010). The Culture of Diagrams. Stanford: Stanford University Press. 
Biggs, Norman, Lloyd, E. Keith, Wilson, Robin J. (1976). Graph Theory 1736-1936. Oxford: Clarendon Press.

Black, M. 1962. Models and Metaphors. Ithaca and New York: Cornell University Press.

Boumans, Marcel (2016). 'Graph-Based Inductive Reasoning', Studies in History and Philosophy of Science vol. 59, pp. 1-10.

Brain, Robert, and Wise, M. Norton (1994). 'Muscles and Engines: Indicator Diagrams in Helmholtz's Physiology', in Lorenz Krüger (ed) Universalgenie Helmholtz: Ruckblicknach 100 Jahren. Berlin: Akademie Verlag, pp. 124-145,

Campbell, Chris (2017). The Chemistry of Relations. The Periodic Table Examined Through the Lens of Charles S. Peirce's Philosophy. PhD Thesis, University College London. Available electronically at: http://discovery.ucl.ac.uk/10039485/1/Chris\%20Campbell\%20Final\%20Thesis\%20Submitted.pdf (last visited 14 August 2019).

Carnap, Rudolf [1932] (1959). 'The Elimination of Metaphysics through Logical Analysis of Language'. Erkenntnis 2, 219-41 (1932) trs. A. Pap. In A.J. Ayer (ed.), Logical Positivism, New York: Free Press, 60-81 (1959)

Coopmans, Catelijne, Vertesi, Janet, Lynch, Michael and Woolgar, Steve (eds.) (2014). Representations in Scientific Practice Revisited. Cambridge, Mass.: The MIT Press.

Corry, Leo (1996). Modern Algebra and the Rise of Mathematical Structures. Basel: Birkhouser. Daston, Lorraine, and Galison, Peter (2007). Objectivity. New York: Zone Books.

Daston, Lorraine (2014), 'Beyond Representation', in Catelijne Coopmans, Janet Vertesi, Michael Lynch, and Steve Woolgar (eds.) (2014). Representations in Scientific Practice Revisited. Cambridge, Mass.: The MIT Press, pp. 319-322.

Dipert, Randall, (1996). 'Reflections on Iconicity, Representation and Resemblance: Peirce's Theory of Signs, Goodman on Resemblance and Modern Philosophies of Language and Mind', Synthese vol. 106 no. 3, pp. 373-397.

Eddy, Matthew (2014). 'How to See a Diagram: A Visual Anthropology of Chemical Affinity', OSIRIS, vol. 29, pp. 178-196.

Galison, Peter (1997). Image and Logic. Chicago: University of Chicago Press.

Giaquinto, Marcus (2005). 'Mathematical Activity', in Paolo Mancosu (ed.), Visualization and Reasoning Styles in Mathematics. Dordrecht: Springer, pp. 75-87.

Giaquinto, Marcus (2007). Visual Thinking in Mathematics, Oxford: Oxford University Press.

Giaquinto, Marcus (2008). 'Visualising in Mathematics', in Paolo Mancosu (ed.) The Philosophy of Mathematical Practice. New York and Oxford: Oxford University Press, pp. 22-42.

Giaquinto, Marcus (2016). 'The Epistemology of Visual Thinking in Mathematics', The Stanford Encyclopedia of Philosophy, Edward N. Zalta (ed.), URL = https://plato.stanford.edu/archives/win2016/entries/epistemology-visual-thinking/ . Last visited 31 July 2019. 
Giardino, Valeria (2013). 'A Practice-Based Approach to Diagrams', in Sun-Joo Shin and Amirouche Moktefi (eds). Visual Reasoning with Diagrams. Basel: Birkhäuser, pp. 135-151.

Giardino, Valeria (2018). 'Tools for Thought: The Case of Mathematics', Endeavour vol. 42, no. 2-3, pp. 172-179.

Giere, Ronald N., (1988). Explaining Science: A Cognitive Approach. Chicago and London: The University of Chicago Press.

Giere, Ronald N., (2004). Scientific Perspectivism. Chicago and London: The University of Chicago Press.

Gooding, David (1990). Experiment and The Making of Meaning. Dordrecht: Kluwer.

Gooding, David (2006). 'From Phenomenology to Field Theory: Faraday's Visual Reasoning', Perspectives on Science, vol. 14, no. 1, pp. 40-65.

Hankins, Thomas (1999). 'Blood, Dirt and Nomograms', ISIS vol. 90 no. 1, pp. 50-80.

Hankins, Thomas (2006). "A Large and Graceful Sinuosity': John Herschel's Graphical Method', ISIS, vol. 97, pp. 605-633.

Hankins, Thomas, and Silverman, Robert J. (1995). Instruments and the Imagination. Princeton: Princeton University Press.

Helmholtz, Hermann von (1850). 'Messungen über den zeitlichen Verlauf der Zuckung animalischer Muskeln und die Fortpflanzungsgeschwindigkeit der Reizimg in den Nerven', Archiv für Anatomie, Physiologie und wissenschaftliche Medicin, 276-381.

Holmes, Frederic, and Olesko, Kathryn (1995). 'The Images of Precision: Helmholtz and the Graphical Method in Physiology', in M. Norton Wise (ed.), The Values of Precision. Princeton: Princeton University Press, pp. 198-221.

Hookway, Christopher (2000). Truth, Rationality and Pragmatism. Themes from Peirce. Oxford: Oxford University Press.

Hookway, Christopher (2012). The Pragmatic Maxim: Essays on Peirce and Pragmatism. Oxford: Oxford University Press.

Jardine, Nick (2012). 'Review of Lorraine Daston and Peter Galison's Objectivity', British Journal of Philosophy of Science, 63 (4), 885-893.

Kaiser, David (2005). Drawing Theories Apart. Chicago: The University of Chicago Press.

Kiryushchenko, Vitaly (2015). ' Maps, Diagrams and Signs: Visual Experience in Peirce's Semiotics', in Peter P. Trifonas (ed.), International Handbook of Semiotics. Dordrecht: Springer, pp. 115-123.

Klein, Judy (1997). Statistical Visions in Time: A history of Time Series Analysis 1662-1938.

Cambridge: Cambridge University Press.

Kramer, Sybille, and Ljungberg, Cristina. 'Thinking in Diagrams. An Introduction', in Sybille Kramer and Christina Ljungberg, Thinking With Diagrams: the Semiotic Basis of Human Cognition. Berlin: De Gruyter, pp. 1-19. 
Kush, Martin (1995). Psychologism: A Case Study in the Sociology of Philosophical Knowledge. London: Routledge.

Kusukawa, Sachiko (2016). 'Classics from this Journal: Martin Rudwick's 'The Emergence of a Visual Language for Geological Science 1760-1840', History of Science vol. 54 no. 1, pp. 98-104.

Latour, Bruno (1990). 'Drawing Things Together', in Michael Lynch and Steve Woolgar (eds), Representation in Scientific Practice, Cambridge, Mass.: The MIT Press, pp. 19-68.

Legg, Cathy (2011). 'The Hardness of the Iconic Must: Can Peirce's Existential Graphs Assist Modal Epistemology?', Philosophia Mathematica vol. 3 no. 00, pp. 1-24.

Legg, Cathy (2013). 'What is a Logical Diagram?' in Sun-Joo Shin and Amirouche Moktefi (eds). Visual Reasoning with Diagrams. Basel: Birkhäuser, pp. 1-18.

Ljungberg, Christina (2015). 'Cartosemiotics', in in Peter P. Trifonas (ed.), International Handbook of Semiotics. Dordrecht: Springer, pp. 759-769.

Lynch, Michael, and Woolgar, Steve, (1990). Representation in Scientific Practice. Cambridge, Mass.: The MIT Press.

Lynch, Michael, 'Representation in Formation', in in Catelijne Coopmans, Janet Vertesi, Michael Lynch, and Steve Woolgar (eds.) (2014). Representations in Scientific Practice Revisited. Cambridge, Mass.: The MIT Press, pp. 323-327.

Maas, Harro and Morgan, Mary (2002). 'Timing History: The Introduction of Graphical Analysis in $19^{\text {th }}$ Century British Economics', Revue d'Histoire des Sciences Humaines, vol. 2 no. 7, pp. 97-107.

Mancosu, Paolo (2005). "Visualisation in Logic and Mathematics", in Paolo Mancosu (ed.), Visualization and Reasoning Styles in Mathematics. Dordrecht: Springer, pp. 13-30.

Meynell, Letitia (2009). 'Why Feynman Diagrams Represent', International Studies in the Philosophy of Science, vol. 22, no. 1, pp. 39-59.

Meynell, Letitia (2018). 'Picturing Feynman Diagrams and the Epistemology of Understanding', Perspectives on Science vol. 26, no. 4, pp. 459-481.

Morgan, Mary (2012). The World in the Model. Cambridge: Cambridge University Press.

Morgan, Mary and Morrison, Margaret (1999). Models as Mediators. Cambridge: Cambridge University Press.

Mumma, John (2010). 'Proofs, Pictures and Euclid', Synthese vol. 175, pp. 255-287.

Peirce, C. (1869). 'The Pairing of the Elements', Chemical News, 4(June), pp. 339-340.

Peirce, C.S. (1879). 'A Quincuncial Projection of the Sphere', American Journal of Mathematics vol. 2, pp. 394-397.

Peirce, Charles Sanders (1931-8/1958). Collected Papers (8 vols.), C. Hartshorne, A. Burks and P. Weiss eds. Cambridge, Mass: Harvard University Press, Cambridge, Massachusetts.

Peirce, Charles Sanders (1982-Present). The Writings of Charles Sanders Peirce: A Chronological Edition. 8 vols. Ed. The Peirce Edition Project. Bloomington, IN: Indiana University Press. 
Peirce, Charles Sanders 1992-98. The Essential Peirce: Selected Philosophical Writings (2 vols.). N. Houser and C. Kloesel eds. (vol.1) and The Peirce Edition Project (vol.2). Bloomington: Indiana University Press.

Perini, Laura (2010). 'Scientific Representations and the Semiotics of Pictures', in P.D Magnus and j. Busch (eds), New Waves in Philosophy of Science. Basingstoke: Palgrave Macmillan, pp. 131-154.

Perini, Laura (2013). 'Diagrams in Biology', Knowledge and Engineering Review, vol. 28 no. 3, pp. 273-286.

Pickstone, John V. (2009). 'The Disunities of Representation', British Journal for the History of Science 42(4), 595-600.

Pietarinen, Ahti-Veikko (2006). Signs of Logic. Dordrecht: Springer.

Pietarinen, Ahti-Veikko (ed.) (Forthcoming). Logic of the Future. Vol. 1, History and Applications. Berlin: De Gruyter.

Pietarinen, Ahti and Bellucci, Francesco (2016a). 'Two Dogmas of Diagrammatic Reasoning. A View from Existential Graphs', in Kathleen A. Hull and Richard Kenneth Atkins (eds.) Peirce on Perception and Reasoning: From Icons to Logic. New York and London: Routledge, pp. 174-195.

Pietarinen, Ahti, and Bellucci, Francesco (2016b). 'The Iconic Moment. Toward a Peircean Theory of Diagrammatic Imagination', in Juan Redmond, Olga Pombo Martins, Ángel Nepomuceno Fernández, (Eds.) Epistemology, Knowledge and the Impact of Interaction. Dordrecht: Springer, pp. 463-481.

Priest, Greg, De Toffoli, Silvia, Findlen, Paula (2018). 'Tools of Reason: The Practice of Scientific Diagramming from Antiquity to the Present', Endeavour vol.42, no. 2-3, pp. 49-59.

Robin, Richard (1967). Annotated Catalogue of the Papers of Charles Sanders Peirce. Worcester: University of Massachusetts Press.

Rudwick, Martin, (1976). 'The Emergence of a Visual Language for Geological Science, 1760-1840', History of Science vol. 14 no. 3, pp. 149-195.

Schmidgen, Henning (2014). The Helmholtz Curves, tr. Nils F. Schott. New York: Fordham University Press.

Sheredos, Ben, Burnston, Daniel, Abrahamsen, Adele, and Bechtel, William (2013). 'Why do Biologists Use so Many Diagrams?', Philosophy of Science vol. 80, no. 5, pp. 931-944.

Shin, Sun-Joo (1994). The Logical Status of Diagrams, Cambridge: Cambridge University Press.

Shin, Sun-Joo (2002). The Iconic Logic of Peirce's Graphs. Cambridge, Mass.: The MIT Press.

Shin, Sun-Joo, Lemon, Oliver, and Mumma, John (2018). 'Diagrams', The Stanford Encyclopedia of Philosophy, Edward N. Zalta (ed.), URL = https://plato.stanford.edu/archives/win2018/entries/diagrams/ . Last Visited 31 July 2019.

Stjernfelt, Frederick (2000). 'Diagrams as a Centrepiece of a Peircean Epistemology'. Transactions of the Charles S. Peirce's Society, vol. 36 no. 3, pp. 357-384.

Stjernfelt, Frederick (2007). Diagrammatology: An Investigation on the Borderlines of Phenomenology, Ontology and Semiotics. Dordrecht: Springer. 
Stjernfelt, Frederick (2011). 'Operational and Optimal Iconicity in Peirce's Diagrammatology', Semiotica vol. 186, pp. 395-419.

Suárez, M. 2010. 'Scientific Representation', Philosophy Compass vol. 5 no.1, pp. 91-101.

Sylvester, Joseph J. (1878). 'On the Application of the New Atomic Theory to the Graphical Representations of the Invariants and Covariants of Binary Quantics - With Three Appendices', American Journal of Mathematics, vol. 1, pp. 64-128.

Tappenden, Jamie (2001). 'Recent work in Philosophy of Mathematics', The Journal of Philosophy vol. 98 no. 9, pp. 488-497.

Teller, Paul, (2001). 'The Twilight of the Perfect Model Model' Erkenntnis 55, 393-415.

Tennant, Neil (1986). 'The Withering Away of Formal Semantics?' Mind and Language, vol. 1 no. 4, pp. 382-318.

Van Fraassen, B. 1980. The Scientific Image. Oxford: Oxford University Press.

Van Fraassen, B. 2008. Scientific Representation. Paradoxes of Perspective. Oxford: Oxford University Press.

Whewell, William (1858). Novum Organon Renovatum. London: John Parker and Son.

Wise, Norton M. (2006). 'Making Visible'. ISIS, vol. 97 no. 1, pp. 75-82.

Wise, Norton M. (2018). 'On the Stories Told by Indicator Diagrams', Endeavour, vol. 42 no. 2-3, pp. 145-156.

Woolgar, Steve (2014). 'Struggles With Representation: Could it be Otherwise?', in in Catelijne Coopmans, Janet Vertesi, Michael Lynch, and Steve Woolgar (eds.) (2014). Representations in Scientific Practice Revisited. Cambridge, Mass.: The MIT Press, pp. 329-332.

Woolgar, Steve, and Lezaun, Javier (2013). 'The Wrong Bin Bag: A Turn to Ontology in Science and Technology Studies?', Social Studies of Science, vol. 43 no. 3, pp. 321-340. 